\title{
Design and Analysis of Materials' Environmental Compatibility
}

\author{
Zeng Cheng ${ }^{1 *}$ \\ ${ }^{1}$ Chongqing College Of Architecture And Technology, Chongqing, 400000, China
}

\begin{abstract}
The material should be in harmony with the environment during its life cycle. This article reviews the origin, concept, research status and application prospects of eco-environmental materials, discusses the environmental coordination evaluation methods of eco-environmental materials, discusses the principles and methods of environmental coordination design of various materials, and lists several typical examples of research on eco-environmental materials. It focuses on the separation and removal of impurities during the alloy regeneration cycle, as well as the organic polymer regeneration cycle technology. In this regard, this article conducts a series of analyses on the development trend of ecological environment materials.
\end{abstract}

\section{Introduction}

Raw materials and manufacturing are the main causes of energy shortages, excessive consumption or depletion of resources, and the rate of consumption is still accelerating. More than $90 \%$ energy and $80 \%$ industrial raw materials come from mineral resources. Every year, more than 5 billion tons of human mineral raw materials are invested, and China's steel output has reached 110 million tons, ranking first in the world. Traditional building materials are mainly cement, glass, ceramics and clay bricks, and the output ranks first in the world. Among them, the annual output of cement reached 560 million tons, accounting for $31 \%$ of the world's total. Energy consumption per 10,000 yuan of GDP is three times the world average and 7.2 times that of Japan. As far as iron and steel metallurgy is concerned, the resource consumption of iron and steel industry is 3.6 times the world average. In 1994, the energy consumption of 11 major raw material industries accounted for $39.97 \%$ of the total industrial energy consumption that year. The waste emissions of the nine major raw material industries accounted for $31.0 \%, 44.5 \%$ and $66.7 \%$ of the industrial waste water, waste gas and solid waste emissions respectively. The average emissions per ton of cement dust and smoke are $23.2 \mathrm{~kg} / \mathrm{t}$, and the annual emissions are $981.21 \mathrm{~kg} / \mathrm{t}$. Material is the material basis and forerunner of social and economic development. Since the beginning of the 290s, in the international research on new materials, a new field has emerged, ecological environmental materials, whose English name is ecomaterials. Sustainability strategy has received extensive attention due to its complexity, multi-level and globalization issues, and more and more attention is paid to the coordination of materials in the environment

\section{Substances are environmental materials}

\subsection{Ecological and environmental material concept}

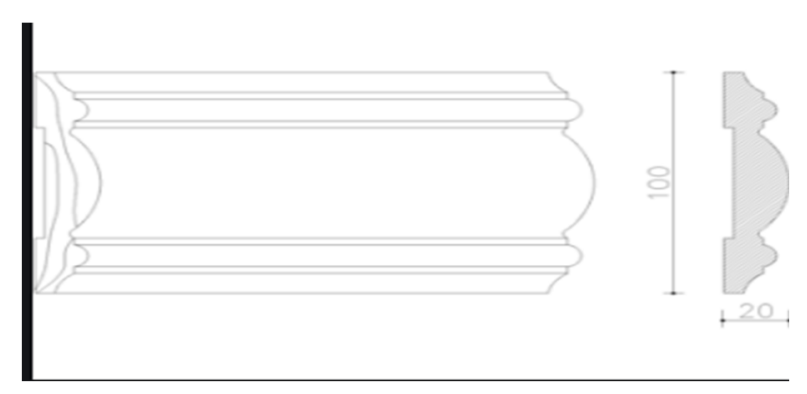

Fig1. Eco-environmental materials

As can be seen from the above picture, the context of the term eco-environmental materials is very complicated. Professor Ryoichi Yamamoto of Japan Future Technology Association put forward the concept of eco-environmental materials in 1990. It means that materials have both good performance and environmental coordination, or can improve the environment. Environmental coordination refers to resource and energy consumption as little as possible, and the impact on the ecological environment is small, and the recycling rate is high. Environmental coordination requires that the entire life cycle from material manufacturing, use to disposal must be in harmony with the environment. Ecological materials refer to traditional structural materials and functional materials. It has special environmental coordination. This is not just a newly developed new material. At the same time, ecological environmental materials also include those that can directly purify and repair the environment.

\footnotetext{
"Corresponding author e-mail: tg778899@xzcstudio.com
} 


\subsection{Coordinated environmental assessment of materials}

\subsubsection{Concept of LCA}

CETAC proposed the official name of LCA in 1990. It was later regulated by the International Organization for Standardization (ISO). ISO9001 Environmental Coordination Assessment (LCA) is defined as the compilation and evaluation of the material energy output and potential environmental impact of a product system throughout its life cycle. The production system is a collection of operation process units with specific functions and related to material energy. Production can refer to both the product system of general manufacturing and the service system of service industry. The life cycle is a continuous and interrelated production stage in the product system from raw material acquisition to the production of human natural resources before the final product is discarded. The idea is to qualitatively and quantify the environmental impact of the product system from the three aspects of energy consumption, human health and ecological environmental impact by collecting environmental catalog data related to the product system and using a set of calculation methods defined by LCA. It can evaluate, and further analyze and look for ways to improve product environmental performance.

\subsubsection{Coordinated environmental assessment of materials}

MLCA is to use the basic concepts, principles and methods of LCA to evaluate the life cycle of materials. In view of the large proportion of environmental pollution caused by materials, it is very important to carry out environmental coordinated evaluation. Standard material evaluation is the basis of many product evaluation, and the repeatability can be reduced by using standard material evaluation to evaluate the promotion material. It can be seen from the following figure.

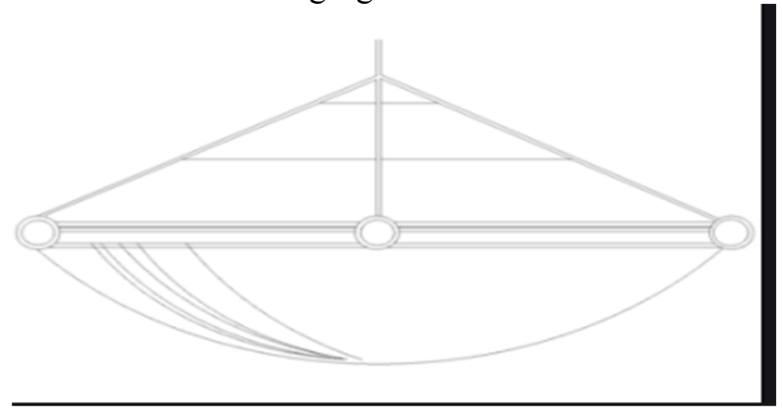

Fig2. Coordinated environmental assessment of materials

\section{Principles and methods for the design of environmentally coordinated materials}

\subsection{Concept}

Conventional material design is mainly based on the development of high-performance materials, resulting in more and more complex material composition, and the possibility of material recycling becoming less and less. Environmentally coordinated design is a new concept produced by applying the LCA method to the material design process. It requires that the design must fully consider factors such as performance, quality, cost and environmental coordination, and put forward material design indicators and suggestions from the perspective of environmental coordination. Environmental coordination design mainly studies the performance evolution mechanism and effect of materials during the regeneration cycle, and harmful impurities removal technology and impurity harmless technology during the regeneration cycle, general alloy technology and material performance prediction technology, etc.

\subsection{General alloy environment coordinated design of main and single alloys}

\subsubsection{General alloy}

The more types of metals, the more difficult it is to recycle. In the best state, all metal products are made of an alloy series. General alloy refers to the alloy system with the lowest element number that can change the material properties in a larger range by adjusting the element. General alloys can reduce the types of product materials. At the same time, a smaller number of elements in the alloy can improve the regeneration cycle performance of the alloy.

\subsubsection{Single alloy}

Single alloy refers to the composition of a single alloy system. The way to change the material properties is to select the appropriate ingredients (no harmful ingredients, no exhausting ingredients) and control the thermal processing process. For example, SCIFER steel is developed by Japan and the separation and harmlessness of impurities during the material regeneration cycle. Currently, in the use of metal materials, steel accounts for more than $90 \%$. The composition of steel contains a variety of alloying elements. In addition, metal products also have metal coatings and non-metallic coatings. There are also several compositions of steel. During the recycling process, the accumulation of $\mathrm{Cu}, \mathrm{Ni}, \mathrm{Sn}, \mathrm{Mo}$ and other elements in the steel increases. As a result, the chemical composition of recycled steel is complex and the quality is reduced. The environmental load in the production process of aluminum products is very large. Energy consumption is 4.5 times higher, and emissions are 7 to 9 times higher. However, the use of waste aluminum for regeneration requires only $3 \%$ of the energy consumption of electrolytic production of primary aluminum. Therefore, when designing aluminum alloy materials, it is important to determine whether the recycling cycle is easy. The main factor hindering lead regeneration is the poor performance of recycled aluminum. Current research hotspots mainly focus on the harmless treatment of Ai-Si alloy impurities. 


\subsection{Design renewable organic polymer recycling}

\subsubsection{Plastic recycling multiple times}

In the recycling process, due to the influence of impurities, aging and processing technology, recycled plastics have the problem of degraded use. Plastic chemical recycling. The plastic chemical recycling is to hydrolyze or depolymerize the recycled plastic, decompose it into monomers or oligomers of the original material, and then synthesize plastic, or decompose the plastic into petroleum. For example, the Plastic Decomposition Fractionation Recovery Technology of the Wire and Cable Research Institute of Japan, the Household Plastic Packaging Material Fractionation and Recovery Technology of Petroleum in Germany, and the Recycling of Kerosene and Diesel Fuel from Waste Plastics of the Hokkaido Institute of Industrial Technology in Japan.

\subsubsection{Recycling design of organic composites}

The plastic is light and soft, but its mechanical properties are low and cannot be made into high-strength structural materials. Inorganic materials, such as glass fiber, carbon fiber have high strength, but poor adhesion and brittleness. Therefore, combining these two materials with the advantages of both can be made into environmentally compatible composite materials. Glass fiber reinforced plastics and carbon fiber reinforced plastics are typical representatives of composite materials. Taking the U.S. HiMAT aircraft as an example. Its GFRP usage is about $4 \%$. Cars that use GFRP as their bodywork have reduced their quality by $51.5 \%$ and greatly reduced fuel consumption. It is expected that the use of CFRP in spacecraft will reach $60 \%$ in the future. The complex is difficult to regenerate. The reason is that during the regeneration, the old materials are crushed and the fibers are crushed and lose their reinforcement. Moreover, after the plastic composite is crushed, the fiber is also crushed and loses the strengthening effect. And plastic alloy can solve this problem. Plastic parts are usually a composite of plastic and liquid crystal polymer.

\section{Various types of eco-environmental materials}

\subsection{Photocatalytic degradation of ecological glass}

Optical degradable glass is a glass with antibacterial and bactericidal effect on pollutants in atmosphere and water under sunlight or ultraviolet radiation. $\mathrm{TiO} 2$ is an excellent material for photocatalytic degradation of glass surface. Film is a kind of photocatalytic degradation and degradable environmental material with great development prospects, but it has not yet been massproduced and applied. The main problem at present is that the efficiency of solar catalysis needs to be further improved. The research on photocatalytic degradation of glass surface pollutants is not comprehensive and in-depth.
There is literature describing instruments for photocatalytic degradation of pollutants such as formic acid, acetic acid and other low-molecular organic compounds. There are few reports about the photocatalytic degradation of polymer organics. Membrane production methods and permeation technology need to be further studied.

\subsection{Expanded graphite}

China is a big country with graphite resources. Expanded graphite is a kind of loose porous wormlike material which is made of natural graphite flake by impregnation, washing, drying and high temperature swelling. It not only retains the natural graphite heat resistance, corrosion resistance, radiation resistance, non-toxic and other excellent properties, but also has the natural graphite without the softness, compression resilience, adsorption performance, ecological environment coordination, biological compatibility and other characteristics. Elastic graphite is a foil made of expanded graphite rolling or pressing, which has excellent sealing performance. In 1978, the domestic flexible graphite sealing material industrialization. Expanded graphite has good biocompatibility and is used in medical dressing instead of gauze. After repeated expansion, the volume can be increased by 300 times to purify the containing SO.. NO. At present, Japan and Israel are working on using expanded graphite to remove oil contamination. For example, the oil and grease pollution in the Three Gorges Reservoir can be removed by adsorption with expanded graphite.

The expansion volume can be increased by 300 times and can be used to purify SO..NO. Japan and Israel are studying the use of expanded graphite to remove oil pollution. For example, the oil and grease pollution of the Three Gorges Reservoir can be absorbed by expanded graphite. The environmental load of manufacturing cement is great. The current research focuses on substitutes of silicate and limestone to reduce the energy consumption of cement burning and reduce $\mathrm{CO} 2$ emissions. For example, Japan's Chibun and Onoda Cement Co., Ltd. use the ash from the incineration of municipal waste and the dewatered sewage sludge as the main raw materials. Its main feature is the direct use of $5 \%-15 \%$ gasification in the ash and slag of municipal waste incineration without treatment. Tongji University has successfully developed a new type of slag cement, which is characterized by high content, high standard and low calorific value.

\subsection{Antibacterial ceramics}

There are three main substances used in ceramics have antibacterial effects metal, they are oxides, silicate carrier metal ions, photocatalytic antibacterial purification materials and so on. 


\subsubsection{Titanium oxide photocatalytic antibacterial ceramics}

The principle of photocatalytic antibacterial method is to use the active oxygen generated by light, which has the functions of purification, autotrophic and antibacterial at the same time, and can be used for a long time. Therefore, these products have broad application prospects in environmental protection. However, previously, when solgel is used to produce ceramics, enamels and glass products, it is difficult to carry out effective control due to the need for special equipment, the product cost was high, and the antibacterial property.

\subsubsection{Antibacterial porcelain}

Silver, copper and other elements are added to the ceramic glaze, and after glazing and sintering, it is uniformly dispersed in the glaze layer on the ceramic surface and exists for a long time. When the addition amount exceeds $3 \%$, the quality of the product is not easy to guarantee, and the production cost is high. When the amount is less than $3 \%$, the dissolution rate of $\mathrm{Ag}^{\prime}$ in the antibacterial glaze is very slow, and it reaches the cell membrane through diffusion, and then is absorbed by the cell membrane. It cannot be metabolized. $\mathrm{Ag}+, \mathrm{Cu} 2+, \mathrm{Zn} 2-$ are toxic to microorganisms (bacteria, molds, viruses, including algae and protozoa), $\mathrm{Ag}+, \mathrm{Cu} 2+, \mathrm{Zn} 2-$ have bactericidal and antibacterial effects on microorganisms, and $\mathrm{Ag}+$ can destroy cell activity. Silver antibacterial ceramic material has good antibacterial effect, safe and reliable. In Japan, the Food Analysis Center conducted bacterial tests on antibacterial ceramic products, and the results were less than $1 \%$ of ordinary ceramic products. Silver-based ceramics with strong antibacterial properties can effectively prevent bacteria from entering the dark and humid, sanitary corners, and can remove dirt and odor in urine.

\section{Conclusion}

However, a unified understanding of the concept of ecoenvironmental materials has not yet been formed. MICA evaluation indicators, evaluation theories, evaluation methods, etc. have not yet formed a system. The coordination between performance and environment is often a contradiction, and it is very difficult to research and develop eco-environmental materials. For the coordinated design of the environment, it is still in the research stage and the relevant theories are not yet mature. Environmental performance is bound to become a basic performance of new materials in the 21st century. MICA technology will also become a conventional evaluation method. Research on the substitution and resource utilization of non-renewable resources will become a hot spot in the materials industry. The development of various eco-friendly materials and their products will become the direction of the development of the materials industry.

\section{References}

1. Shi Xiaohong, Li Jun, Sang Xuemei. The design of materials for environmental coordination[J]. Chongqing Environmental Science, 2003, 25(007): 54-57.

2. Wang Wei. Research on the design and development direction of garden architectural sketches[J]. Modern Horticulture, 2013(08):78.

3. Shen Wen. Comprehensive atrium landscape evaluation and application research from the perspective of landscape architecture[D]. 2019. 